\title{
Squeezing Millimeter Waves into Microns
}

\author{
Alastair P. Hibbins and J. Roy Sambles \\ Thin Film Photonics Group, School of Physics, University of Exeter, Stocker Road, Exeter EX4 4QL, United Kingdom
}

Christopher R. Lawrence and James R. Brown

QinetiQ Ltd., 1146/A7, Cody Technology Park, Ively Road, Farnborough GU14 OLX, United Kingdom

(Received 25 November 2003; published 9 April 2004)

\begin{abstract}
Microstructured metallic devices will play a vital role in the continuing search to manipulate the passage of electromagnetic radiation relevant to optical, microwave, and communication technologies. Here, we investigate the electromagnetic response of a completely novel and ultrathin (« wavelength) structure within which is buried a metal-clad waveguiding layer ("core") of subwavelength width. By removing metal from the core cladding to form a periodic array of slits, radiation is coupled into a standing wave within the layer and the structure resonantly absorbs or transmits radiation of wavelength more than 100 times its thickness. Additionally, such structures display the truly remarkable capability of compressing half of the standing-wave wavelength into a fraction of the expected distance.
\end{abstract}

DOI: 10.1103/PhysRevLett.92.143904

The discovery that metals perforated with arrays of subwavelength apertures can transmit more radiation than impinges directly upon the voids [1] has sparked a wealth of work to understand the underlying physics and develop potential applications (for example, Refs. [214]). Studies of the aforementioned transmission phenomena have thus far been restricted to single layer structures. Here we explore the electromagnetic response of structures formed from a closely spaced pair of parallel metal layers, at least one of which is perforated with an array of slits of subwavelength width.

While the intriguing observations of Ebbesen et al. [1] were obtained using metal films perforated with twodimensional arrays of cylindrical holes, many subsequent studies have instead addressed the problem of onedimensional arrays of slits. Multiple diffraction is commonly regarded to be responsible for the "anomalous" results observed from both hole and slit arrays; importantly, however, the means by which the mechanism is resonantly enhanced differs [2-9]. For example, the transmission channel through relatively thin metal films relies on the evanescent mode within the apertures, and the surface plasmon polariton [15] which propagates on the faces of the array plays an important role [3,5$10,12,13]$. However, according to classical waveguide theory [16], narrow slits (but not holes) are able to support TEM waveguide modes that have no cutoff in frequency. An open-ended slit of length $L$ will support a series of Fabry-Perot-like standing-wave modes associated with the impedance mismatch of the entrance and exit apertures. Resonance of these modes thereby provides a strong enhancement to the multiple diffraction mechanism that is not available to hole arrays $[3,4,8,11]$. Peaks in the transmission spectra are therefore exhibited according to the equation

$$
f_{N} \approx N c / 2 n L,
$$

PACS numbers: 42.25.Bs, 42.79.Dj, 73.20.Mf, 84.40.-x

where $f_{N}$ is the resonant frequency on the $N$ th order Fabry-Perot mode, $c$ is the vacuum speed of light, and $n$ is the refractive index of the dielectric filling the cavity. Consider a sample in the form of two thin metallic layers of high conductivity (to maintain opaqueness to radiation) and forming a regular array of long, narrow, and parallel slots all the way though one or both of the layers [Fig. 1(a)]. It will be shown here that radiation incident upon one of the metal faces, and polarized

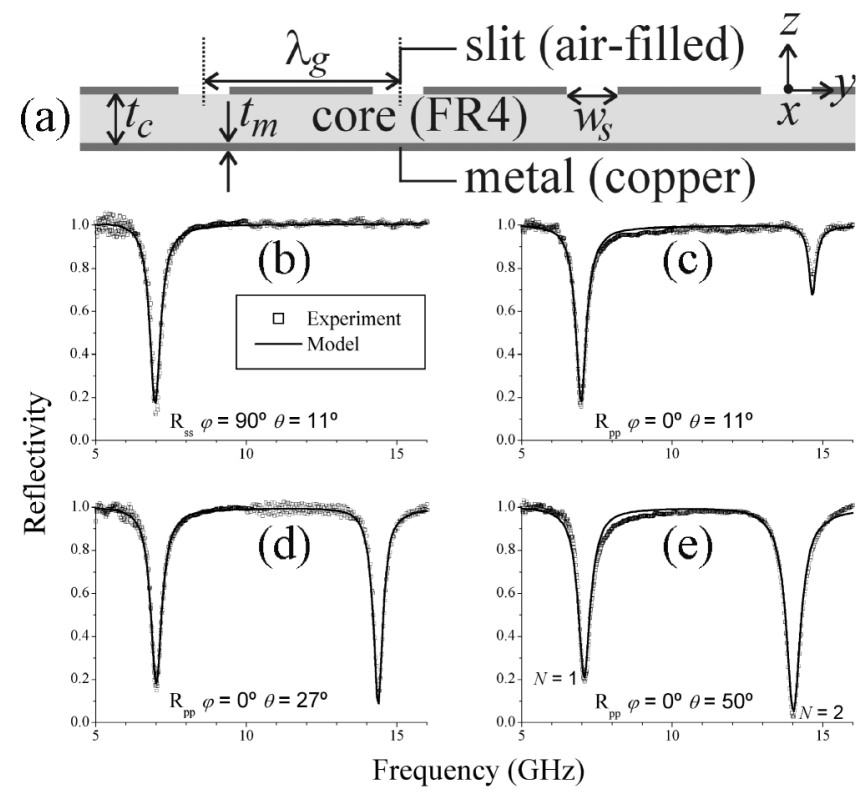

FIG. 1. Experimental reflectivity spectra and the numerically modeled results from the structure illustrated schematically in (a). (b) The $R_{s s}$ response of the structure when orientated with the slit direction parallel to the plane of incidence $\left(\varphi=90^{\circ}\right)$. (c)-(e) The $R_{p p}$ response with $\varphi=0^{\circ}$ at three different angles of incidence. The change in noise at $10 \mathrm{GHz}$ corresponds to a switch in the set of waveguides and horn antennas used. 
with a component of its electric vector orthogonal to the slits, will couple through the gaps, and excite a standing-wave mode within the metal-clad core. Hence, the resonant frequencies of the modes supported by these extremely thin devices (of thickness $\sim \lambda_{0} / 100$ ) are quantized by the pitch of the slot array.

Samples are made using standard "print and etch" techniques [17] from printed circuit boards (PCB) of area approximately $500 \mathrm{~mm} \times 500 \mathrm{~mm}$ with an epoxy glass-cloth laminated core (FR4) of thickness, $t_{c}=$ $356 \mu \mathrm{m}$ bounded by $18 \mu \mathrm{m}\left(=t_{m}\right)$ of copper cladding. Initially studies are performed on a structure that has slots etched into only one face of the PCB [Fig. 1(a)], with a pitch of $10 \mathrm{~mm}\left(\lambda_{g}\right)$ and slit widths of approximately $0.3 \mathrm{~mm}\left(w_{s}\right)$. A planar microwave beam of frequency $5 \leq$ $f \leq 16 \mathrm{GHz}$ is incident upon the sample at a fixed polar angle $(\theta)$. The chosen pitch of the slits dictates that no propagating diffracted orders emerge from the structure within the studied frequency range for $\theta<60^{\circ}$.

Figure 1(b) illustrates the frequency-dependent response for $\theta=11^{\circ}$ with the sample orientated so that the slits are parallel, and the electric vector perpendicular to the plane of incidence $\left(\varphi=90^{\circ}, s\right.$ polarized $)$. The minimum at approximately $7 \mathrm{GHz}$ is due to absorption of energy on resonance of a standing wave within the core. Equation (1) provides an estimation of the frequency of this mode with the length of the core, $L$ substituted for the pitch, $\lambda_{g}=10 \mathrm{~mm}$, and also setting $N=1$ and $n(\mathrm{FR} 4) \approx 2.05$ [18]. Excitation of this mode in the dielectric layer would be expected to correspond to high electric fields, of opposite sign, underneath adjacent slits with one nodal plane midway between the slits. Hence, with $s$-polarized radiation incident at any $\theta$, and $\varphi=90^{\circ}$, no reflectivity minimum should be observed since the phase of the incident electric field at every slit is identical. Similarly, one expects strong coupling to the $N=2$ mode as this should require identical electromagnetic phase at each slit. However, the experimental results shown in Fig. 1(b) tell a very different story. We investigate the coupling conditions and electromagnetic field solutions by comparing the predictions from a finite element method (FEM) [19] model to our experimental observations, and it transpires that they are dramatically different from those naively anticipated.

The solid lines on the graphs of Fig. 1 are the predictions from the FEM model, where the slit width, pitch, and permittivity of the core have been used as fitting parameters, and the values used for the core and copper thickness are as specified by the PCB manufacturer ( $t_{c}=$ $0.356 \mathrm{~mm}$ and $t_{m}=0.018 \mathrm{~mm}$, respectively). The best fits are achieved with pitch, $\lambda_{g}=10.030 \mathrm{~mm}$ and slit width, $w_{s}=0.350 \mathrm{~mm}$, which agree with the values measured from the device using a traveling microscope $\left(\lambda_{g}=\right.$ $10.03 \pm 0.03 \mathrm{~mm}$ and $w_{s}=0.35 \pm 0.01 \mathrm{~mm}$ ). In addition, the fitted value of the core permittivity (FR4), $\varepsilon=$ $4.17+0.07 i$, accords well with that determined by others
[18]. Using these parameters, the electric field solution is calculated on resonance of the $N=1$ standingwave mode at $7.0 \mathrm{GHz}$, and is shown in Fig. 2(a). Surprisingly, a region of zero field strength is exhibited on the lower copper layer directly beneath the slit. This is because the incident electric field induces charges of opposite sign in the metal on either side of the slit width, hence requiring the phase of the standing-wave field within the core to be completely reversed $(\pi$ phase change) as the width of the slit is traversed [Figs. 2(b) and 2(c)]. Hence, the truly remarkable aspect of this structure is that, while half of the standing wave is sustained within the region of the core bounded by the continuous region of the copper layers, the remaining half of the cycle, corresponding to another $10 \mathrm{~mm}$, is compressed

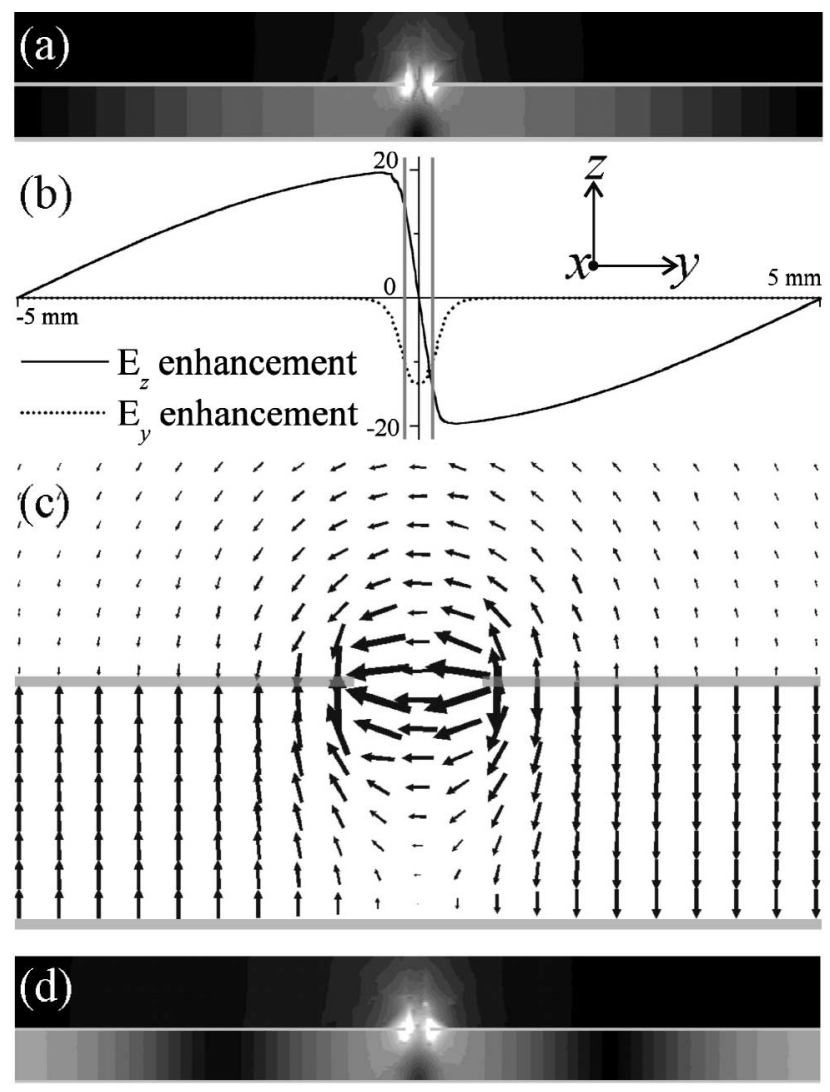

FIG. 2. (a)-(c) Electric field enhancement throughout the structure on resonance of the $N=1$ mode $(7 \mathrm{GHz})$ visible in Fig. 1(e). (a) The time-averaged field magnitude where white regions represent enhancements of at least 40 times. The region of the structure shown corresponds to a unit cell. (b),(c) The instantaneous fields at a phase corresponding to the strongest enhancement. (b) illustrates the $y$ and $z$ components along a line at the midheight of the core, where the grey vertical lines correspond to the edges of the slit. (c) A vector representation of the field distribution in a magnified region around the slit. (d) The time-averaged electric field magnitudes associated with the resonance of the $N=2$ mode at $14.05 \mathrm{GHz}$ with $\theta=$ $50^{\circ}$ [Fig. 1(e)]. White regions represent field magnitude enhancements of at least 20 times. 
into a length of the order of the slit width $(\sim 0.5 \mathrm{~mm})$. Note that this half-wave compression is so strong that it does not significantly perturb the quantization condition giving the mode frequencies.

As a consequence of this addition of $\pi$ radians of phase to the standing-wave solution, and contrary to expectations, coupling to the $N=1$ mode is permitted at normal incidence. Similarly, the compression of half the standing wave into almost zero distance changes the coupling condition of the $N=2$ mode so as to require opposite phases at adjacent slits. Hence, with $s$ polarization, no reflectivity minimum is observed at $\sim 14 \mathrm{GHz}$. However, with $p$-polarized radiation incident and the slits orientated so that they lie perpendicular to the plane of incidence $\left(\varphi=0^{\circ}\right)$, any deviation away from $\theta=0^{\circ}$ creates a phase shift along the surface and coupling to the even $N$ harmonics becomes possible [Figs. 1(c)-1(e)].

To further unravel this phenomenon, the transmission spectra of two further structures $(d=0 \mathrm{~mm}$ and $d=$ $5 \mathrm{~mm}$ ) were studied, but now with slits in both upper and lower metal surfaces. Microwave radiation of frequency $5 \leq f \leq 30 \mathrm{GHz}$ is incident normally upon each sample $\left(\theta=0^{\circ}\right)$, orientated so that the slit direction is perpendicular to the electric field vector. The experimentally detected transmissivities are shown in Fig. 3. The best fits (solid lines) were obtained using the FEM model with $\lambda_{g}=10.020$ and $w_{s}=0.290 \mathrm{~mm}$ for the $d=0 \mathrm{~mm}$ structure and $\lambda_{g}=10.010$ and $w_{s}=0.328 \mathrm{~mm}$ for the $d=5 \mathrm{~mm}$ structure (compared to measured values of $\lambda_{g}=10.02 \pm 0.02$ and $w_{s}=0.29 \pm 0.02 \mathrm{~mm}$, and $\lambda_{g}=$ $10.01 \pm 0.01$ and $w_{s}=0.33 \pm 0.02 \mathrm{~mm}$, respectively). In agreement with our results discussed above, the per-

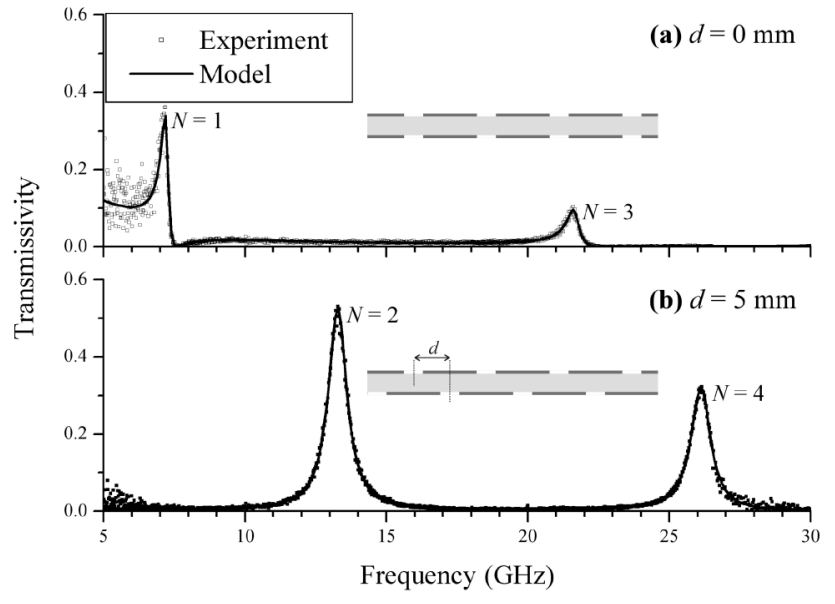

FIG. 3. Experimental frequency-dependent transmissivity data from (a) a structure whose slits on either side of the core are directly opposite each other $(d=0 \mathrm{~mm})$, and (b) a structure with $d=\lambda_{g} / 2=5 \mathrm{~mm}$. Also shown are the numerically modeled results. Radiation is normally incident $\left(\theta=0^{\circ}\right)$ and detected, and the sample is orientated so that the slit direction is perpendicular to the electric field vector. mittivity of the core which gives the best comparison is $\varepsilon=4.17+0.07 i$.

Interestingly, on resonance of the $N=1$ mode $(f=$ $7.2 \mathrm{GHz}$ ) of the $d=0 \mathrm{~mm}$ structure, the electric field at the upper slit is completely out of phase with that at the lower aperture and there is a region of zero electric field approximately at the slit center at the midheight of the core [Figs. 4(a) and 4(b)]. The phase change of the standing waves measured over the region bounded by continuous metal is $N \pi$, with an additional $\pi$ radians across the slit width. Therefore only coupling to the odd $N$ harmonics is possible at normal incidence. However, with $\theta \neq 0^{\circ}$,
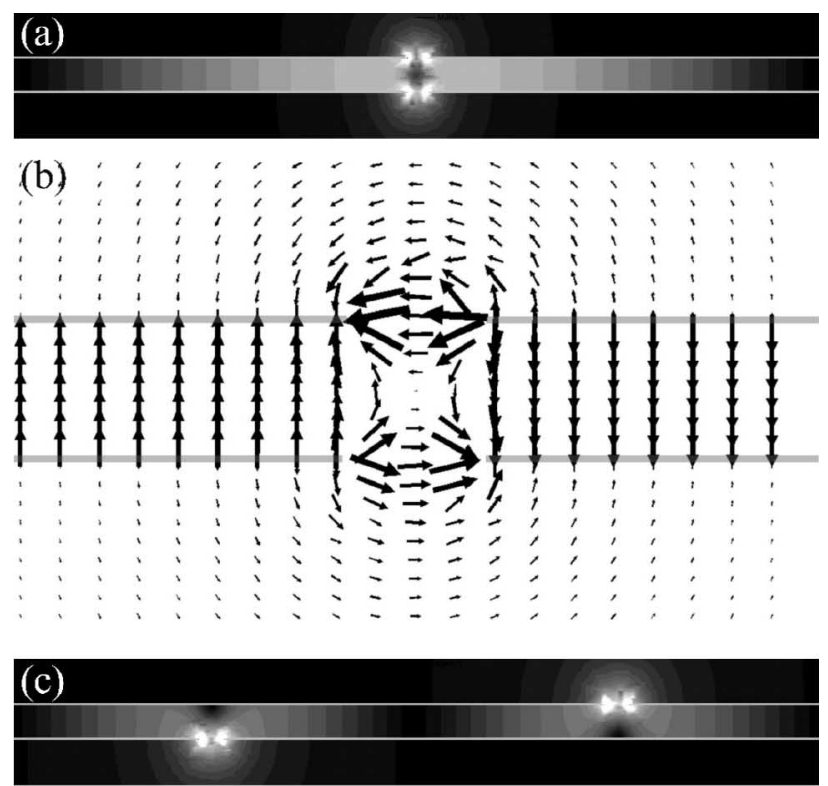

(d)

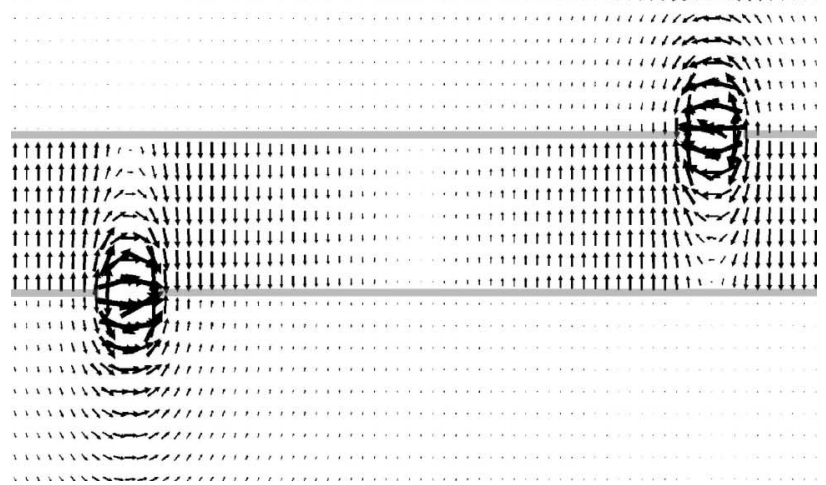

FIG. 4. Electric field enhancement throughout the two transmitting structures on resonance of each of their lowest frequency modes. (a),(b) $N=1, d=0 \mathrm{~mm} f=7.2 \mathrm{GHz}$; (c), (d) $N=2 d=\lambda_{g} / 2=5 \mathrm{~mm}, f=13.3 \mathrm{GHz}$. The grey scale plots, (a) and (c), illustrate the time-averaged field magnitudes, plotted over an entire unit cell (pitch) of the sample, where white regions correspond to enhancements of 20 times or more. Parts (b) and (d) show the instantaneous vector field distribution in magnified regions around the slits at a phase corresponding to the strongest enhancement. 
the phase change of the incident field along the surface enables even harmonics to be excited. Now consider the structure with the lower slits displaced in the $y$ direction by $d=\lambda_{g} / 2=5 \mathrm{~mm}$. This separation of the upper and lower slits means that the addition of $\pi$ radians of phase occurs twice - at both the upper and lower slits. Hence, coupling to the even $N$ modes is now possible at all angles of incidence, e.g., $N=2$ [Figs. 4(c) and 4(d)]. What about the odd $N$ harmonics? For resonant transmission to occur, regions of high electric field intensity must exist directly below the slits in the illuminated surface and above the slits in the lower metal layer. Clearly, these two conditions cannot both be satisfied for odd $N$ harmonics. Perhaps one would therefore expect it to absorb radiation on resonance of the odd $N$ modes, corresponding to regions of high electric field underneath the illuminated slits and high magnetic field (and low electric field) above the lower slits? However, the break in the lower metal surface positioned midway between the upper slits forbids this solution for any angle of incidence. It blocks surface currents, preventing the required region of high magnetic field.

The increase in off-resonance transmissivity through the symmetric structure with decreasing frequency is another intriguing phenomenon [Fig. 3(a)]. The structure is acting similar to a low-pass frequency selective surface [20] where the excitation of the standing-wave resonances perturbs the response that would otherwise be expected. Additionally, there is a frequency just above each mode at which the intensity falls to zero. The reason for this is simple. As previously discussed, on resonance of a standing wave within the core, there exists a region of zero field intensity between the slits, approximately at the midheight of the core. As the frequency is increased, this node moves towards the lower slit, until it is located at the lower aperture. At this point, the magnitude of the transmitted signal is zero. Further increase in frequency results in strengthening fields at the lower slit, which are now in phase with those at the upper slit, and a transmitted signal.

In conclusion, we have presented an experimental and modeling study of the electromagnetic response of a series of ultrathin, metal-clad waveguiding structures, formed by simply removing metal from the cladding, giving a periodic array of subwavelength slits. Astonishingly, these devices are able to resonantly absorb or transmit radiation of wavelength some 100 times greater than their thickness via coupling of energy into a standing wave within a common core. In addition, we have revealed their ability to compress the $\pi$ phase of the standing wave directly beneath the slit, effectively squeezing millimeters into microns. All these features make such structures excellent candidates for ultrathin absorbers, radiators, and filters. In addition, the remarkable phase compression will have direct consequences for future electromagnetic technologies.

This work was carried out as part of Technology Group 09 of the MoD Corporate Research program. The authors also wish to acknowledge the financial support of the EPSRC.

[1] T.W. Ebbesen, J. J. Lezec, H. F. Ghaemi, T. Thio, and P. A. Wolff, Nature (London) 391, 667 (1998).

[2] M. M. J. Treacy, Phys. Rev. B 66, 195105 (2002).

[3] J. A. Porto, F. J. García-Vidal, and J. B. Pendry, Phys. Rev. Lett. 83, 2845 (1999).

[4] E. Popov, M. Nevière, S. Enoch, and R. Reinisch, Phys. Rev. B 62, 16100 (2000).

[5] A. Krishnan et al., Opt. Commun. 200, 1 (2001).

[6] S. Salomon, F. Grillot, A.V. Zayats, and F. de Fornel, Phys. Rev. Lett. 86, 1110 (2001).

[7] L. Martín-Moreno et al., Phys. Rev. Lett. 86, 1114 (2001).

[8] S. Collin, F. Pardo, R. Teissier, and J.-L. Pelouard, J. Opt. A 4, S154 (2002).

[9] M. Sarrazin, J.-P. Vigneron, and J.-M. Vigoureux, Phys. Rev. B 67, 085415 (2003).

[10] Q. Cao and P. Lalanne, Phys. Rev. Lett. 88, 057403 (2002).

[11] S. Astilean, Ph. Lalanne, and M. Palamaru, Opt. Commun. 175, 265 (2000).

[12] J. Gómez Rivas, C. Schotsch, P. Haring Bolivar, and H. Kurz, Phys. Rev. B 68, 201306 (2003).

[13] W. L. Barnes et al., Phys. Rev. Lett. 92, 107401 (2004).

[14] B. Schechter, New Sci. 178, 30 (2003).

[15] W. L. Barnes, A. Dereux, and T.W. Ebbesen, Nature (London) 424, 824 (2003).

[16] R. E. Collin, Field Theory of Guided Waves (IEEE, New York, 1991), 2nd ed.

[17] Eurotech Group Plc., Exmouth, UK.

[18] E. Kemppinen, Doctoral dissertation, University of Oulu, 1999 (herkules.oulu.fi/isbn9514251954/).

[19] HFSS, Ansoft Corporation, Pittsburgh, PA, USA.

[20] B. A. Munk, Frequency Selective Surfaces, Theory and Design (Wiley, New York, 2000). 